\title{
Correlation between Nucleon-Nucleon Interaction, Pairing Energy Gap and Phase Shift for Identical Nucleons in Nuclear Systems
}

\author{
Willy K. Koech*, Ken M. Muguro, Godfrey S. Murunga ${ }^{\circledR}$, Kapil M. Khanna \\ Department of Physics, University of Eldoret, Eldoret, Kenya \\ Email: *willykipronokoech@gmail.com, mwauraken@yahoo.com, muguro@uoeld.ac.ke, murungagodfrey12@gmail.com, \\ khannak700@gmail.com
}

How to cite this paper: Koech, W.K. Muguro, K.M., Murunga, G.S. and Khanna, K.M. (2019) Correlation between Nucleon-Nucleon Interaction, Pairing Energy Gap and Phase Shift for Identical Nucleons in Nuclear Systems. Journal of High Energy Physics, Gravitation and Cosmology, 5, 321-331.

https://doi.org/10.4236/jhepgc.2019.52018

Received: December 25, 2018

Accepted: February 17, 2019

Published: February 20, 2019

Copyright $\odot 2019$ by author(s) and Scientific Research Publishing Inc. This work is licensed under the Creative Commons Attribution International License (CC BY 4.0).

http://creativecommons.org/licenses/by/4.0/

\begin{abstract}
Assuming some known nucleon-nucleon interactions, and using the relations between phase shift $\delta$ and nucleon-nucleon interaction potential $V(r)$; the relation between nucleon-nucleon interaction and scattering length $a$; the relation between energy gap $\Delta$, and scattering length $a$; an equation is obtained between energy gap $\Delta$ and Fermi momentum $k_{F}$ via the phase shift $\delta\left(k_{F}\right)$. Assuming ${ }^{1} \mathrm{~s}_{0}$ (singlet) pairing between the nucleons, the energy gap $\Delta$ has been calculated and it is found that $\Delta=3.0 \mathrm{MeV}$ at Fermi momentum $k_{F}=0.8 \mathrm{fm}^{-1}$.
\end{abstract}

\section{Keywords}

Scattering Length, Pairing Gap, Phase Shift, Fermi Momentum

\section{Introduction}

When one particle approaches another particle, and they are in the field of force of each other; they interact and scatter. In this process the following three parameters are involved.

1) The interaction potential $V(r)$ between the two particles;

2) The distance between the nearest-approach, called impact parameter or the scattering length $a$;

3) Phase shift, $\delta$, due to scattering.

However, when dealing with large finite-nuclei, or infinite nuclear matter and neutron matter (in stars), another important parameter gets involved, and this is the so called ${ }^{1} s_{0}$ pairing gap $\Delta$. Thus there must exist, a definite relationship 
between the interaction potential $V(r)$. The scattering length $a$, the phase shift $\delta$ and the pairing gap $\Delta$. Thus the quantitative features of ${ }^{1} \mathrm{~s}_{0}$ pairing in nuclear matter and neutron matter can be obtained directly from the ${ }^{1} s_{0}$ phase shifts. The ${ }^{1} s_{0}$ neutron matter superfluid is relevant for the phenomena that occur in the inner crust of neutron stars [1]. The ${ }^{1} s_{0}$ pairing gap values and its density dependence show a peak value of about $3 \mathrm{MeV}$ at a Fermi momentum close to $k_{F} \cong 0.8 \mathrm{fem}^{-1}$ [2]; most of the calculations adopt the bare nucleon-nucleon interaction as the pairing force and it has been pointed out that the screening by the medium of interaction could strongly reduce the pairing strength in this channel [3].

After the discovery of neutron stars calculations were done for pairing gap for neutrons [4] in the ${ }^{1} s_{0}$ state. The pairing gap energy for neutrons [4] in the ${ }^{1} s_{0}$ state rose with increasing density and reached a maximum of roughly $3 \mathrm{MeV}$ at the density of about $n=n_{s} / 10$, where $n_{s}=0.16 \mathrm{fm}^{-1}$ is the saturation density of symmetric nuclear matter (containing equal numbers of neutrons and protons), a density typical of the interiors of heavy nuclei. With further increase in density, the neutron pairing energy gap dropped and vanished at a density just below $n_{s}$ (i.e. $n$ has less than $n_{s}$ ). At such density, it becomes favorable for neutrons to pair in the ${ }^{3} \mathrm{P}_{2}$ state $(2 s+1=3, l=1, s=1, J=l+s=1+1=2)$ in which the pairs have unit orbital angular momentum $(l=1)$, unit spin $(s=1)$ and total angular momentum $(J=l+s=2)$. It should be emphasized that the ${ }^{3} \mathrm{P}_{2}$ state has more attractive interaction than the other ${ }^{3} \mathrm{P}$ sates due to the fact that the spin-orbit interaction is attractive for nucleons. However in atomic physics the spin-orbit interaction is repulsive. For the neutron pair in the state ${ }^{3} \mathrm{P}_{2}$, the pairing energy gap is increased to about $0.5 \mathrm{MeV}$ at a density of around $2 n_{s}$ and dropped at higher densities. The qualitative behavior of the pairing energy gaps is understood in terms of the measured phase shifts for nucleon-nucleon interactions. A positive phase shifts corresponds to an attractive interaction between neutrons and therefore at low $k$ ( $k$ is the propagation vector $p / \hbar$ ), which corresponds to low Fermi momentum and low density. The most attractive is channel is ${ }^{1} \mathrm{~S}_{0}$, which at higher densities the interaction in ${ }^{3} \mathrm{P}_{2}$ channel is more attractive and hence the phase shift will be positive.

In BCS approximation, the pairing energy gap is calculated by solving the BCS equation with the free-space nucleon-nucleon interaction and free particles in intermediate states in the scattering process; the effects of the neutron medium on the normal state excitations and the pairing interaction are neglected [5] [6]. Many different techniques have been developed to include effects beyond the BCS approximation in the calculations of neutron pairing energy and most of them that the pairing gap reduces by a factor of 2 or more. However microscopic calculations have been for pairing energy gap for nuclear and neutron matter in the $1 \mathrm{~S} 0$ state ${ }^{3} \mathrm{p}_{2}$ state and ${ }^{1} \mathrm{~S}_{0}$ state for protons. The pairing gap $\Delta$ has also been related to the nucleon-nucleon interaction and phase shift.

Going by BCS approximation and considering pairing in the ${ }^{1} \mathrm{~S}_{0}$ state, the gap 
is independent of the direction of $k$ and the relation between gap $\Delta(k)$ and the interaction potential $v(r)$ is given by [7]

$$
\Delta(k)=-\frac{1}{\pi} \int_{0}^{\infty} \delta k^{\prime} k^{\prime} \frac{V\left(k, k^{\prime}\right)}{E\left(k^{\prime}\right)} \Delta\left(k^{\prime}\right)
$$

where $V\left(k, k^{\prime}\right)$, is the matrix element of the potential averaged over the angle between $k$ and $k^{\prime}$ given by

$$
V\left(k, k^{\prime}\right)=\int_{0}^{\infty} \delta r r^{2} j_{0}\left(k^{\prime} r\right) V(r) j_{0}(k r)
$$

We can use two values for $V(r)$ one is the Yukawa potential, i.e.

$$
V(r)=-V_{0}\left(\frac{b}{r}\right) e-\frac{v}{b}
$$

where

$$
b=2 \mathrm{fm} \text { and } V_{0}=30 \mathrm{MeV}
$$

Another is the simple Gaussian potential of the form,

$$
V(r)=-V_{0} \mathrm{e}^{-\alpha r^{2}}
$$

where

$$
V_{0}=5037.0 \mathrm{MeV} \text { and } \alpha=12.0 \mathrm{fm}^{-2}
$$

This potential has been used in the phase shift analysis of the ${ }^{2} \mathrm{~S}_{1 / 2}$ scattering phase. Substituting the values of $V(r)$ from Equation (3) and Equation (5) in Equation (2) and then substituting the values of $V\left(k, k^{\prime}\right)$ in Equation (1) we can get the relation between $\Delta(k)$ and $V_{0}$ clearly emphasizing of the there exists a close relationship between the pairing energy gap and the interaction potential. A rough calculations assuming $E\left(k^{\prime}\right) \approx \Delta\left(k^{\prime}\right)$ leads a constant-value for $V\left(k, k^{\prime}\right)=-\frac{v_{0}}{b}$ and substituting this in Equation (1) will give a finite value of $\Delta(k)$ assuming some finite limits for integral in Equation (1), similarly we can use the value of $V(r)$ in Equation (5) and obtain the value of $\Delta(k)$. Hence there exists a definite correlation between the pairing energy gap $\Delta(k)$ and obtain the interaction potential. Without making any approximations, exact value of $\Delta(k)$ can also be calculated [8].

\section{Theoretical Derivations}

The nuclear force has been at the heart of nuclear physics since the discovery of the neutrons by Chadwick [9]. The interaction between two nucleons is basic for all of nuclear physics. The main aim of nuclear physics is to understand the properties of atomic nuclei in terms of the "bare" interaction between a pair of nucleons. Scattering of nucleons is due to the neutrons neutrons-interaction between the nucleons and hence the resulting phase shifts will have a definite correlation with the interaction potential $v(r)$.

From time to time, a nuclear of nucleon-nucleon interaction potentials has 
been proposed. For instance, Yukawa potential [10] is the oldest attempt to explain the nature of the nuclear forces. According to Yukawa massive bosons (mesons) mediate the interaction between two nucleons it is given by

$$
v(r)=\frac{\beta}{r} \mathrm{e}^{-\frac{r}{\beta}}\left(-v_{0}\right)
$$

Another potential is a simple Gaussian potential of the form [11] [12].

$$
v(r)=v_{0} \mathrm{e}^{-\alpha r^{2}}
$$

where $v_{0}=5037.0 \mathrm{MeV}, \alpha=12.0 \mathrm{fm}^{-2}$

In the last few decades the major issues concerning the (nucleon-nucleon) interaction have been:

1) Charge-dependence;

2) The precise value of the $\pi$ nucleon-nucleon compiling constant;

3) Improved phase shift analysis;

4) High precision nucleon-nucleon data;

5) High-precision nucleon-nucleon potentials;

6) Quantum-chromo-Dynamics (QCD) and the nuclear force;

7) Nuclear-nuclear scattering is at intermediate and high energies.

However, in this manuscript we are interested in some simple calculations that will correlate the well known nucleon-nucleon interaction potential $v(r)$ with the phase shift $\delta$ the scattering length $a$ and the energy gap $\Delta$; and the dependence of $\Delta$ on the Fermi momentum $k_{F}$ has also been studied. Calculations have been done using Yukawa potential only.

Using Yukawa potential calculations are done to relate interaction potential $V(r)$ to the phase shift $\delta_{l}(k)$ and energy gap $\Delta\left(k_{F}\right)$ Yukawa potential $V(r)=\frac{\beta}{r} \mathrm{e}^{-\frac{r}{\beta}}$ where $V(r)$ the interaction is potential, $r$ is the inter-particle distance, $\beta$ is the range of nucleon-nucleon force and $V_{0}$ is potential well depth.

The Born approximation [13] is a relationship between phase shifts, $\delta_{l}(k)$ and interaction potential $(V(r))$

$$
\delta_{l}=-\frac{2 \mu k_{f}}{\hbar^{2}} \int_{0}^{\infty} V(r) j_{l}^{2}\left(k_{f} r\right) r^{2} \mathrm{~d} r
$$

Using Bessel function in form

$$
j_{0}^{2}\left(k_{f} r\right)=\sin ^{2}\left(\frac{\left(k_{f} r\right)}{\left(k_{f} r\right)^{2}}\right), \text { for } l=0, \text { ground state, where } k_{f} r<1
$$

Using derived equations and the values of the constants available, data was then generated and tabulated. Graphs have been drawn to show how the phase shifts $\delta_{t}\left(k_{F}\right)$, varies with the Fermi momentum $k_{F}$. This potential is substituted in the Born-approximation phase shifts, $\delta_{p}\left(k_{F}\right)$, for scattering from a spherical potential, $V(r)$, in 3-D, and to find the values of phase shifts. 
The pairing gap for small values of $k_{F}\left|a_{0}\right|$ is [14]

$$
\Delta\left(k_{f}\right)=\frac{8}{\mathrm{e}^{2}} \lambda \exp \left(-\frac{\pi}{2 k_{f}\left|a_{0}\right|}\right)
$$

where $a_{0}$ the scattering length in the ISO channel is $\left(a_{0}=-19.3 \mathrm{fm}\right), \lambda$ is a constant $\approx 1, \mathrm{e}=2.718$, here $a_{0}$ is related to the interaction potential between a pair of nucleons. However at saturation density $\rho_{0}=0.17 \mathrm{fm}^{-3}, k_{F}=1.36 \mathrm{fm}^{-1}$.

For low energy scattering especially in nuclear force which corresponds to the size of the potential and $\delta_{t}\left(k_{F}\right)$, due to scattering is given by relation

$$
k_{F} \cot \delta_{\rho}\left(k_{F}\right)=-\frac{1}{a_{0}}+\frac{1}{2} r_{0} k_{F}^{2}
$$

here $r_{0}$ is the effective range of the nuclear force which roughly corresponds to the size of the potential and $\delta_{l}\left(k_{F}\right)$ is the S-wave scattering phase-shift.

At the ground state $\ell=0$ therefore Equation (9) becomes,

$$
\delta_{\ell}\left(k_{F}\right)=-\frac{2 u k_{f}}{\hbar^{2}} \int_{0}^{\infty} V(r) j_{0}^{2}\left(k_{F} r\right) r^{2} \mathrm{~d} r
$$

Here $j_{0}\left(k_{F} r\right)$ is the spherical Bessel function of Zeroth order i.e. $\ell=0$ such that,

$$
j_{0}^{2}\left(k_{F} r\right)=\frac{\sin ^{2}\left(k_{F} r\right)}{\left(k_{F} r\right)^{2}}
$$

It is valid to represent the interaction energy of a particle with momentum $k_{i}<k_{F}$, with all the particles within the Fermi surface. The values for $k_{i}$ [15] could be $0.1 \mathrm{fm}^{-1}, 0.2 \mathrm{fm}^{-1}, 0.3 \mathrm{fm}^{-1} \ldots$ and therefore for $k_{F} r<1$ hence $\sin ^{2}\left(k_{F} r\right) \cong\left(k_{F} r\right)^{2}$ therefore Equation (13) reduces to

$$
j_{0}^{2}\left(k_{F} r\right)=1
$$

Substituting the value of the potential, $V(r)=V_{0} \frac{\beta}{r} \exp ^{-\frac{r}{\beta}}$ in Equation (12) we get

$$
\delta_{0}\left(k_{F}\right)=\frac{2 \mu k_{f} V_{0}}{\hbar^{2}} \int_{0}^{\infty} \frac{\beta}{r} \mathrm{e}^{-\frac{r}{\beta}} r^{2} \mathrm{~d} r
$$

Integrating Equation (15) by parts we get,

$$
\delta_{0}\left(k_{F}\right)=\frac{\mu k_{f} V_{0}}{\hbar^{2} \beta^{2}}
$$

Now for Yukawa potential, the value of the well-depth parameters $S$ is

$$
s=0.17291 \frac{M V_{0}}{\hbar^{2} \beta^{2}}
$$

where $M$ is the average mass of the two interacting nucleons $S \cong 1$ for the bound state of the nuclear matter and hence $V_{0}$ is given by

$$
V_{0}=5.7834 \frac{\hbar^{2} \beta^{2}}{M}
$$


Substituting Equation (17) in Equation (16) gives,

$$
\delta_{0}\left(k_{F}\right)=2.7834 \frac{\mu k_{F}}{M}
$$

For ${ }^{1} \mathrm{~S}_{0}$ scattering and the reduced mass $\mu$ of the two interacting nucleons is given by

$$
\mu=\frac{m_{p} \cdot m_{n}}{m_{p}+m_{n}}
$$

where $m_{p}$ the proton is mass equal to $938.27 \mathrm{meV}$, and $m_{n}$ is the neutron mass equal to $939.57 \mathrm{meV}$. Substituting the values of the masses in Equation (20) reduced mass $\mu=469.46 \mathrm{meV}$. The average mass $M$ of the proton and neutron is given by the equation,

$$
M=\frac{m_{p}+m_{n}}{2}
$$

Substituting for the value of the masses $M=938.92 \mathrm{meV}$, then Equation (19) becomes

$$
\delta_{0}\left(k_{F}\right)=1.1462 k_{F}
$$

Calculating the values of phase shift, $\delta_{0}\left(k_{F}\right)$, for different values of $k_{F}$ ranging from $0.1 \mathrm{fm}^{-1}, 0.2 \mathrm{fm}^{-1}, 0.3 \mathrm{fm}^{-1}, \cdots, 1.6 \mathrm{fm}^{-1}$. And plot a graph of phase shift $\delta_{0}\left(k_{F}\right)$, against Fermi momentum $k_{F}$.

For scattering length in the ${ }^{1} \mathrm{~S}_{0}$ channel $a_{0}=-19.3 \mathrm{fm}, \mathrm{e}=2.718$ and $\lambda \approx 1$ Equation (11) for energy gap [14], $\Delta\left(k_{F}\right)$, becomes

$$
\begin{gathered}
\Delta\left(k_{F}\right)=\frac{8}{2.718^{2}} \cdot \exp \left(\frac{-\pi}{2 \times k_{F} \times-19.3}\right) \\
\Delta\left(k_{F}\right)=\frac{8}{2.718^{2}} \exp \left(\frac{-0.08122}{k_{F}}\right)
\end{gathered}
$$

Equation (23) will give the values of the energy gap.

It will be interesting to see that if other potentials are used for instance the potential used Hassan and Ramadan study [15] how phase shift $\delta_{\ell}\left(k_{F}\right)$ and energy gap $\Delta\left(k_{F}\right)$ vary with Fermi momentum $k_{F}$. For low energy scattering the phase shift is given by $\delta_{\ell}\left(k_{F}\right)$ for different values of the Fermi momentum $k_{F}$.

Singlet scattering length Equation [16] is given by

$$
a_{n n}=\frac{\pi}{2 k_{0 F}}\left(\frac{\sqrt{2 m \epsilon_{c}}}{\hbar k_{0 F}}+\frac{2}{f_{e x}^{\varepsilon}}\right) \equiv \frac{\pi}{4 k_{0 F}}\left(\frac{1}{f_{e x}^{\varepsilon}}-\frac{1}{f_{c r}^{\varepsilon}}\right)^{-1}
$$

where critical constant $f_{c r}^{\varepsilon}=-\frac{2 k_{0 F}}{k_{o c}} \cong-1.912$ and $f_{e x}^{\varepsilon}$ is the vacuum strength.

The Pairing Gap equation is given by

1) At very low density [17]

$$
\Delta=c \epsilon_{F} \exp \left(\frac{\pi}{2 k_{F} a_{n n}}\right) \text { for } a_{n n}<0
$$


where $c=8 \mathrm{e}^{-2}, k=k_{F}$ and $\epsilon_{F}=\frac{\hbar^{2} k_{F}^{2}(\rho)}{2 m}$.

2) At low density [18]

$$
\Delta=\frac{\hbar^{2}}{m}\left(\frac{2 \pi \rho}{a_{n n}}\right)^{\frac{1}{2}}, \text { for } a_{n n}>0
$$

where: $m$ is the free nucleon mass.

\section{Results and Discussion}

Recent studies have shown the nuclear isotope shifts, the differential observables such as the odd-even mass differences and odd-even effects in charge radii along isotope chains can be reproduced with an effective density-dependent contact pairing interaction. The self-consistent LEDF calculations with density gradient term $\alpha f_{\nabla}^{\varepsilon}$ in pairing force provide desirable size of isotopic shifts. Using Lead isotopes some sets of parameters are deduced for the pairing force. Calculations are done based on the general variation-principle applied to local effective density-dependent function with a fixed energy cutoff $\epsilon_{c}=40 \mathrm{MeV}$ measured from $\epsilon_{F}$ and on the coordinate-space technique which involves an interaction [19]. At very low densities

$$
a_{n n}=\frac{\pi}{2 k_{0 F}}\left(\frac{\sqrt{2 m \epsilon_{c}}}{\hbar k_{0 F}}+\frac{2}{f_{e x}^{\varepsilon}}\right) \equiv \frac{\pi}{4 k_{0 F}}\left(\frac{1}{f_{e x}^{\varepsilon}}-\frac{1}{f_{c r}^{\varepsilon}}\right)^{-1}
$$

where critical constant $f_{c r}^{\varepsilon}=-\frac{2 k_{0 F}}{k_{o c}} \cong-1.912$ and $f_{e x}^{\varepsilon}$ is the vacuum strength,

$$
\Delta=c \epsilon_{F} \exp \left(\frac{\pi}{2 k_{F} a_{n n}}\right), \text { for } a_{n n}<0
$$

where $c=8 \mathrm{e}^{-2}, k=k_{F}$ and $\epsilon_{F}=\frac{\hbar^{2} k_{F}^{2}(\rho)}{2 m}$.

These results agree with the general analysis of the gap equation at low densities for $a_{n n} \ll 1$. It is valid only in the weak coupling regime which corresponds to negative scattering length (Figure 1).

At $f_{e x}^{\varepsilon}>f_{c r}^{\varepsilon}$ from Equation (1) it shows that at low densities the pairing gap is small and it comes to zero.

Relation between the phase shift $\delta_{l}$, the nucleon-nucleon interaction $V(r)$ and the energy gap using Yukawa potential:

Using Equation (22) to calculate the values of phase shift $\delta_{0}\left(k_{F}\right)$, for different values of $k_{F}$ ranging from $0.1-1.6 \mathrm{fm}^{-1}$ and plot a graph of phase shift $\delta_{0}\left(k_{F}\right)$, against Fermi momentum $k_{F}$.

When the Fermi momentum $k_{F}$ of the interacting nucleons is zero, the value of phase shift $\delta_{0}\left(k_{F}\right)$, is equal to zero in the ground state as seen from Figure 2, this show that an increase in Fermi momentum $k_{F}$ leads to an increase in phase shift $\delta_{0}\left(k_{F}\right)$.

Using Equation (23) to compute the values of energy gap $\Delta\left(k_{F}\right)$ against 


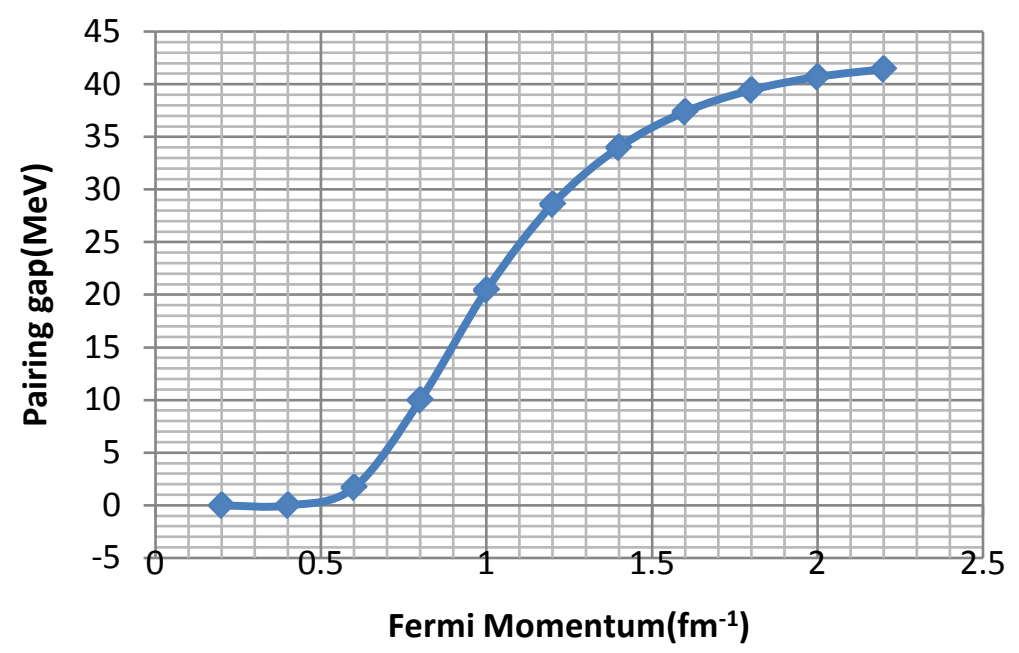

Figure 1. A graph of pairing gap against scattering length for $a_{n n}<0$.

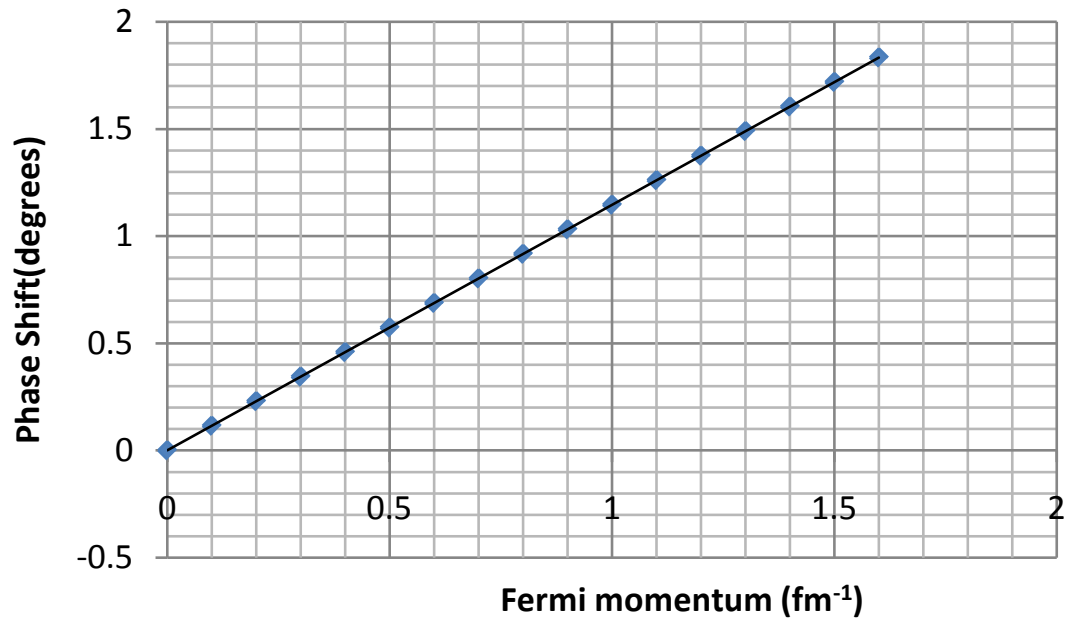

Figure 2. A graph of pairing gap against Fermi momentum $\left(\mathrm{fm}^{-1}\right)$.

changes in the Fermi momentum $k_{F}$ and this variation is done and the data tabulated (Figure 3).

The energy gap $\Delta\left(k_{F}\right)$ increase steadily and faster for low Fermi momentum $k_{F}$ up to around $1.0 \mathrm{fm}^{-1}$ and it is roughly constant with the value $\cong 0.8$ $\mathrm{MeV}$ for $k_{f}>1.0 \mathrm{fm}^{-1}$. In literature the values of the energy gap $\Delta\left(k_{f}\right)$ are $0.4 \mathrm{MeV}, 0.6 \mathrm{MeV}, 0.7 \mathrm{MeV} \ldots$ for $0.2 \mathrm{fm}^{-1}, 0.3 \mathrm{fm}^{-1}, 0.4 \mathrm{fm}^{-1} \ldots$ respectively [15] which are in agreement with the known values.

The energy gap $\Delta\left(k_{F}\right)$ increase steadily and faster for low Fermi momentum $k_{F}$ up to around $0.4 \mathrm{fm}^{-1}$ and it is roughly constant with the value $\cong 1.0$ $\mathrm{MeV}$ for $k_{f}>0.4 \mathrm{fm}^{-1}$. In literature the values of the energy gap $\Delta\left(k_{F}\right)$ are $0.2 \mathrm{MeV}, 0.4 \mathrm{MeV}, 0.6 \mathrm{MeV} \ldots$ for $0.2 \mathrm{fm}^{-1}, 0.3 \mathrm{fm}^{-1}, 0.4 \mathrm{fm}^{-1} \ldots$ respectively [15] which are in agreement with the known values. The reason for the good agreement in that, for calculating energy gaps the quantity that matters is the scattering length at energies of order of the Fermi momentum and this is strongly constrained by nucleon-nucleon scattering data for nucleon momentum in the 


\section{A graph of energy gap against Fermi momentum}

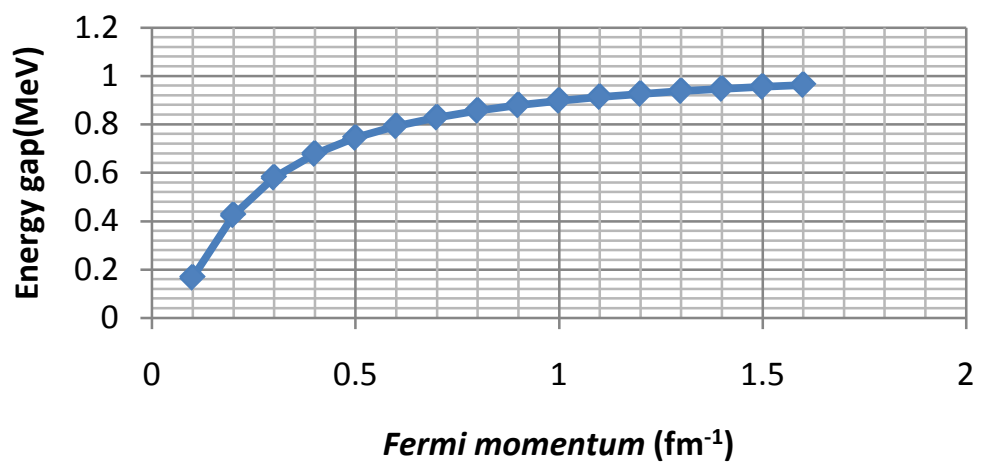

Figure 3. A graph of energy gap against Fermi momentum $\left(\mathrm{fm}^{-1}\right)$.

center-of-mass. For higher Fermi momenta the energy gap tends to reach saturation since there is considerable model dependence also because inelastic channels start to open up in nucleon-nucleon scattering. The energy gap $\Delta\left(k_{F}\right)$ increases steadily and faster for low values of Fermi momentum $k_{F}$ and becomes roughly constant for values of $k_{F}>0.4 \mathrm{fm}^{-1}$.

\section{Conclusion}

From the results obtained, it can be concluded that scattering length influences the energy gap with different values of Fermi momentum. The behavior of pairing gap $(\Delta)$ at very low densities agrees well with calculations based on realistic nucleon-nucleon forces. The singlet scattering length which is $a_{n n}=-19.3 \mathrm{fm}$ agrees with the theoretical value which is equivalent to $a_{0}=-18.3 \mathrm{fm}$. Numerical values of scattering length are negative for $k_{F}<2 \mathrm{fm}^{-1}$ which means attractive force and positive for $k_{F}>2 \mathrm{fm}^{-1}$ indicating repulsive force. At low densities the pairing effect is affected by strong repulsive and short-range component interaction; therefore, the predictions are set to go higher for pairing gap $(\Delta)$ reaching a maximum of $1.69 \mathrm{MeV}$ at $k_{F}=0.4 \mathrm{fm}^{-1}$ with nucleon-nucleon interaction assuming charge dependence. Interactions between nucleons may be characterized by a single parameter, the S-wave scattering length $a_{n n}$ which indicates the strength of interactions. The sign determines whether the interactions are effectively attractive or repulsive. When the scattering length is $a_{n n}<0$ the interaction is attractive and repulsive for $a_{n n}>0$. The behavior of pairing gap $(\Delta)$ at very low densities agrees well with calculations based on realistic NN forces. The singlet scattering length which is $a_{n n}=-20.4 \mathrm{fm}$ at $k_{F}=1 \mathrm{fm}^{-1}$ agrees with the theoretical value which is equivalent to $a_{n n}=-18.3 \mathrm{fm}$. Numerical values of scattering length are negative for $k_{F}<2 \mathrm{fm}^{-1}$ which means attractive force and positive for $k_{F}>2 \mathrm{fm}^{-1}$ indicating repulsive force. When the Fermi momentum $k_{F}$ of the interacting nucleons is at zero the value of phase shift $\delta_{0}\left(k_{F}\right)$, is equal to zero in the ground state as seen from Figure 2. This show that, increase in Fermi momentum $k_{F}$ leads to an increase in phase shift 
$\delta_{0}\left(k_{F}\right)$.

\section{Suggestions}

In future this problem can be done using nucleon interactions that may involve elementary particles. But such calculations will be quite complicated and will require the use of many-body techniques involving Greens functions can be done. Recent discoveries on elementary particles have led to the suggestions for different types of nuclear interactions involving many parameters. Elementary particles inside the nucleus were the neutrons and protons. But within the theory of quantum chromo-dynamics (QCD), neutrons and protons are no longer the elementary particles. Nuclear forces between neutrons (neutron-neutron force). Protons (proton-proton force) and neutron-proton (neutron-proton force) were treated as charge independent. Within the Theory of QCD, there is what is called Charge Symmetry Breaking (CSB) and consequently the neutron-neutron-scattering length, the proton-proton-scattering length and neutron-proton scattering length change. Consequently there is a corresponding phase shift variation with $a_{n n}$, $a_{p p}$ and $a_{n p}$, and there is a different relation with the nuclear force. These calculations can be done in the future.

\section{Acknowledgements}

We are grateful to our colleagues at the Department of Physics, University of Eldoret for their helpful discussions, contributions and suggestions during the preparation and writing of this research paper. We also appreciate the international Centre of theoretical physics for their eJDS platform for providing us with several articles and useful information in line with this publication.

\section{Conflicts of Interest}

The authors declare no conflicts of interest regarding the publication of this paper.

\section{References}

[1] Maghdi, M. (2014) Physics of Particles and Nuclei Letters. Physics of Particles and Nuclei Letters, 4.

[2] Elgoray, Q. and Hjorth-Jensen, M. (1997) Nucleon-Nucleon Interaction. Physical Review C, 57, 1174.

[3] Chem, M. (2008) Review of Particle Physics. Physics Letters B, 667, 1-6. https://doi.org/10.1016/j.physletb.2008.07.018

[4] Holfberg, M., Glassgold, A.E., Richardson, R.W. and Ruderman, M. (1970) Anisotropic Superfluidity in Neutron Star Matter. Physical Review Letters, 24, 775. https://doi.org/10.1103/PhysRevLett.24.775

[5] Lombardo, U. and Scheelze, H.J. (2001) Superfluidity in Neutron Star Matter. In: Blaschke, D., Glendenning, N.K. and Sedrakian, A., Eds., Physics of Neutron Star Interiors, Lecture Notes in Physics 578, Springer-Verlag, Berlin, Heidelberg, 30-53. https://doi.org/10.1007/3-540-44578-1_2 
[6] Dean, D.J. and Hjorth-Jeusen, M. (2003) Pairing in Neclear Systems; from Neutron stars to Finite Nuclei. Reviews of Modern Physics, 75, 607. https://doi.org/10.1103/RevModPhys.75.607

[7] Gezerlis, A., Pethick, C.J. and Schwenk, A. (2015) Pairing and Superfluidity of Nucleons in Neutron Stars. arxiv:1406.6109.

[8] Khanna, K.M. (2008) Superconducivity. Moi University, Kesses.

[9] Chadwick, J. (1932) The Existence of a Neutron. Proceedings of the Royal Society of London, A136, 692. https://doi.org/10.1098/rspa.1932.0112

[10] Yukawa, H. (1935) On the Interaction of Elementary Particles I. Proc. Phys. Math. Soc. Jap., 17, 48-57.

[11] Machleidt, R. (1989) The Meson Theory of Nuclear Forces and Nuclear Structure. Advances in Nuclear Physics, 19, 189-376. https://doi.org/10.1007/978-1-4613-9907-0_2

[12] Coon, S.A. and Niskanen, J.A. (1996) Conclusive Evidence for the Influence of Nuclear Orientation on Quasi-Fission. Physical Review C, 53, 1154.

[13] Schiff, L.I. (1968) Quantum Mechanics. Mcgraw-Hill Book Company, New Delhi, 330.

[14] Khanna, K.M. and Barhai, P.K. (1975) Single-Particle Potential, Re-Arrangement Energy and Effective Mass of Nuclear Matter, ${ }^{208} \mathrm{~Pb}$ and ${ }^{16} \mathrm{O}$. Nuclear Physics A, 243, 298-308. https://doi.org/10.1016/0375-9474(75)90249-3

[15] Chadan, K. and Sabatier, P.C. (1992) Inverse Problems in Quantum Scattering Theory. Springer, New York, 105.

[16] Huang, K. (1975) Statistical Mechanics. Wiley Eastern, New Delhi, 37.

[17] Kodel, V.A., Kodel, V.V. and Clark, J.W. (1996) Solution of the Gap Equation in Neutron Matter. Nuclear Physics A, 598, 390-417. https://doi.org/10.1016/0375-9474(95)00477-7

[18] Keldysh, L.V. and Kozlov, A.N. (1968) Collective Properties of Excitons in Semiconductors. Soviet Physics JETP, 27, 521.

[19] Fayans, S.A., Tolokonnikov, S.V., Trykov, E.L. and Zawischa, D. (1998) Nuclear Isotope Shift within the Local Energy-Density Functional Approach. Nuclear Physics $A, 676,49-119$. 